\title{
Comparison of the Effect of Thermal Aging on Dielectric Properties of Vegetable Oils and Impregnated Kraft Paper
}

\author{
Cristina Méndez Gutiérrez \\ Electrical and Energy Engineering \\ Department \\ University of Cantabria \\ Santander, Spain \\ mendezc@unican.es
}

\author{
Hikaru Araki \\ Electrical and Electronic \\ Engineering Department \\ Kyushu Institute of Technology \\ Kitakyushu, Japan
}

\author{
Taichi Yamada \\ Electrical and Electronic \\ Engineering Department \\ Kyushu Institute of Technology \\ Kitakyushu, Japan
}

\author{
Motoo Tsuchie \\ Electrical and Electronic \\ Engineering Department \\ Kyushu Institute of Technology \\ Kitakyushu, Japan
}

\author{
Masahiro Kozako \\ Electrical and Electronic \\ Engineering Department \\ Kyushu Institute of Technology \\ Kitakyushu, Japan
}

\author{
Masayuki Hikita \\ Electrical and Electronic \\ Engineering Department \\ Kyushu Institute of Technology \\ Kitakyushu, Japan
}

\author{
Alfredo Ortiz Fernández \\ Electrical and Energy Engineering \\ Department \\ University of Cantabria \\ Santander, Spain
}

\begin{abstract}
In this paper, dielectric properties (BDV and permittivity) of two vegetable oils (Palm Fatty Acid Ester and FR3) used in power transformers are studied. Also, partial discharge inception voltage (PDIV) of Kraft paper impregnated with these oils is measured. All the tests were carried out at room temperature and the samples were prepared following the same procedure. Both the impregnated paper and the vegetable oils were aged at $140^{\circ} \mathrm{C}$ for 11 days to study the evolution of the dielectric properties with the thermal aging. PDIV has been also measured for different number of paper sheets (from 4 to 6), analyzing the dependence of PDIV on the thickness of solid insulation. Dielectric characterization of the oils showed that they are suitable to use in power transformers. BDV of the oils decreased with the aging and PFAE performance was better. Dielectric constant of both oils is almost the same. PDIV results showed that both oils are suitable for the impregnation of Kraft paper in terms of dielectric properties. PDIV of FR3 insulating system was higher than PFAE system in all the studied cases. FR3 insulating systems showed a decrease of PDIV value after the aging process, whereas PFAE one remained almost constant.
\end{abstract}

\section{INTRODUCTION}

Mineral oil is commonly used as dielectric and coolant liquid in power transformers due to its good properties. During the operation of these devices an important amount of heat is generated in the windings due to the Joule losses. It is necessary to control the temperature of the windings since it is directly related to the transformer lifespan. The fluid cools the transformer and, at the same time, acts as electrical insulation medium.

Despite having good dielectric and thermal properties, in the last years there has been an increase in the interest to substitute mineral oil with new biodegradable fluids. The main reasons are the low biodegradability of mineral oil and the risk of fire in power transformers due to its low fire point.

These new liquids are vegetable base and their properties depend on the origin. Different fluids (palm, soybean, sunflower or rapeseed oils) have been studied [1]. All of them are biodegradable and, except for the palm base oil, their main advantage is the high fire point while the drawback is their high viscosity, which limits their cooling capacity. This is the reason why most of the works are focused on the study of the thermal properties of these new liquids. Less researchers have studied the dielectric properties of these oils and even less the effect of aging on them. Works about dielectric properties of natural esters and their comparison with mineral oil have conflicting results. While $Q$. Liu et al. [2] studied BDV with different test methods and found that mineral oil always has a higher BDV than the natural esters tested, other authors like D. Divakaran and C. Kalaivanan [3] found that mineral oil has worst dielectric properties. The large amount of oils available and the influence of ambient conditions on dielectric response make it difficult to compare the different works.

More research is needed about thermal aging effect on the dielectric properties of different vegetable oils. 
It is also necessary to consider the interaction of the transformer oil with the solid insulation (cellulose). The impregnation process of the cellulose with the transformer oil and its dielectric properties are crucial for the proper operation of the device. Despite its importance, few works have been done with the new liquids. Some of them, as the works developed by J.Dai and Z.D.Wang [4], are focused on the time required for the impregnation process with biodegradable oils in comparison with the mineral oil. The results found that at certain temperature the viscosity and capillary action of ester oils are similar to that of mineral oil. Some works, as the one developed by A. Cavallini et al. [5], study PD of celluloses impregnated with mineral oil, using different electrode configurations to analyze PD patterns. Other few works study dielectric properties with natural esters. A. Prevost [6] and D. Martin et al. [7] analyzed the dielectric properties (BDV, dielectric constant and loss factor) of different celluloses impregnated with mineral oil and natural ester. Results showed better dielectric behavior of vegetable oil insulation system. Research works on the effect of aging on dielectric properties of oil-impregnated paper are scarce.

Based on the above, this paper presents an experimental study on the evolution of dielectric properties of two vegetable oils with the thermal aging. AC Breakdown Voltage (BDV) and permittivity of FR3 and PFAE oils has been measured. Permittivity of both oils has been tested at room temperature before and after the aging process. Also, impregnation with the vegetable oils and thermal aging of Kraft paper was carried out. AC Partial Discharge Inception Voltage (PDIV) has been studied and its dependence on thermal aging and paper thickness has been analyzed.

\section{EXPERIMENTAL PROCEDURE}

\section{A. Materials and preparation of the samples}

High viscosity soybean oil FR3 and Low viscosity Palm Fatty Acid Ester (PFAE) have been used as liquid dielectrics. Their main properties are shown in TABLE I.

The Kraft paper thickness is $200 \mu \mathrm{m}$ and its properties are summarized in TABLE II.

No treatment was applied to the oils before the characterization of their dielectric properties. Moisture content was measured before each test to ensure similar conditions.

TABLE I.

MAIN PROPERTIES OF THE TESTED OILS.

\begin{tabular}{|c|c|c|}
\hline Property and standard & PFAE & FR3 \\
\hline Density at $15^{\circ} \mathrm{C}\left[\mathrm{g} / \mathrm{cm}^{3}\right]$ & 0.8632 & 0.923 \\
\hline Kinetic viscosity at $40^{\circ} \mathrm{C}\left[\mathrm{mm}^{2} / \mathrm{s}\right]$ & 5.062 & 32.19 \\
\hline Fire point by $\mathrm{COC}\left[{ }^{\circ} \mathrm{C}\right]$ & 206 & 356 \\
\hline Flash point by $\mathrm{COC}\left[{ }^{\circ} \mathrm{C}\right]$ & 188 & 326 \\
\hline Breakdown voltage by IEC $60247(\mathrm{kV})$ & 85 & 69 \\
\hline Permittivity at $90^{\circ} \mathrm{C}$ by IEC 60247 & 2.85 & 2.86 \\
\hline Biodegradation by $\mathrm{OECD} 301[\%]$ & 77 & 100 \\
\hline
\end{tabular}

TABLE II.

POPERTIES OF THE KRAFT PAPER

\begin{tabular}{|c|c|}
\hline Property & Specification \\
\hline Apparent density $\left[\mathrm{g} / \mathrm{cm}^{3}\right]$ & 0.754 \\
\hline Moisture $[\%]$ & 6.3 \\
\hline Conductivity of the aqueous extract $[\mathrm{mS} / \mathrm{m}]$ & 1.5 \\
\hline $\mathrm{pH}$ & 6.5 \\
\hline Dielectric strength in air $[\mathrm{kV} / \mathrm{mm}]$ & 8.9 \\
\hline
\end{tabular}

For the preparation of the solid insultation, Kraft paper was cut in $80 \times 80 \mathrm{~mm}$ sheets, according to the size of the electrodes used. Paper sheets were dried in an oven at $100^{\circ} \mathrm{C}$ for 24 hours. Then, they were placed in an iron vessel, where both the impregnation and aging processes were carried out. Moisture content of the oils was measured before the impregnation process using the automatic potentiometric titrator AT-710. It was fixed a maximum moisture content in the new oils of $100 \mathrm{ppm}$, that was fulfilled without any treatment. The oils were degassed before the impregnation process. In order to avoid the moisture absorption a vacuum system was used during this process, connecting a vacuum pump to the iron vessel and the degasser. Kraft paper was totally immersed in the oil for 12 hours in a nitrogen atmosphere.

Accelerated thermal aging of the impregnated paper immersed in oil was carried out in an oven at $140^{\circ} \mathrm{C}$ for 11 days.

Appearance of the oils and impregnated paper before and after the aging process is shown in Fig. 1.

\section{B. Dielectric characterization and PD tests}

It was decided to measure the dielectric properties of the oils to analyze their suitability as dielectric liquids for power transformers. After that, impregnation and aging process of Kraft paper allowed us to study the effect of each fluid on the PDIV value of the insulation system.

AC Breakdown voltage (BDV) of the oils was measured using a $200 \mathrm{ml}$ oil cup with two $2.5 \mathrm{~mm}$ diameter sphere electrodes. BDV of both new and aged samples was determined at three different gap lengths $(0.8,1.6$ and 2.5 $\mathrm{mm}$ ). Voltage was applied using a power transformer connected to a current source controlled with an oscilloscope. Voltage was increased gradually from $0 \mathrm{~V}$ to BDV, with a maximum value of $100 \mathrm{kV}$. Frequency of analysis was $60 \mathrm{~Hz}$. Before the first measurement, the oil was leave in the oil cup for 5 minutes to dissipate bubbles. Between each measurement a pause of 2 minutes was applied. According to IEC 60247 standard, 6 measurements were made for each sample.

A frequency analysis of permittivity was conducted using a Hioki LCR Meter IM3536. The oil was put into the electrode system and the tests were carried out at room temperature for new and aged samples. 


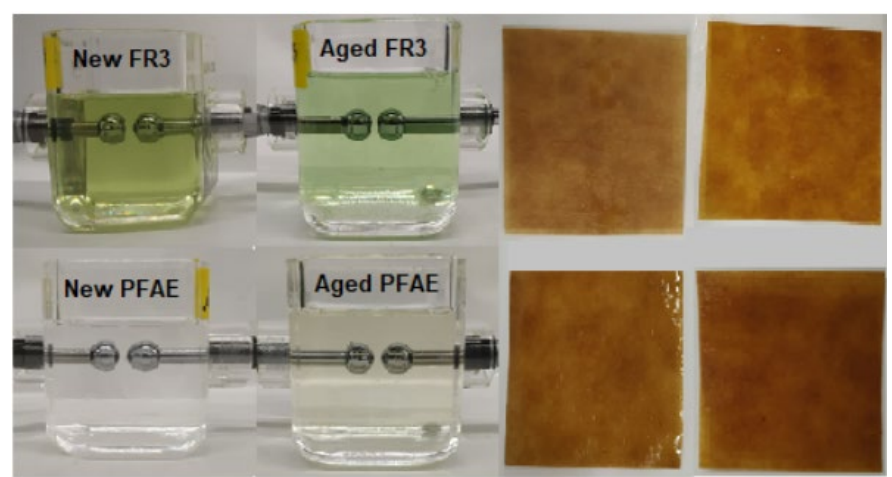

Fig. 1. FR3, PFAE and impregnated paper before and after the aging process.

AC Partial Discharge Inception Voltage (PDIV) of the oilimpregnated papers was measured immediately after removing the samples from the vessel, both for fresh and aged samples. Non-symmetrical plane-to-plane electrode configuration was used. High voltage electrode is a cylindrical electrode while low voltage electrode is a disc type electrode, with a higher diameter. Paper sheets are placed between the electrodes and the system is immersed in the oil. Before performing the PD tests, the samples were degassed in a vacuumed container to dissipate the bubbles that could have appeared between the paper sheets.

PD detector and PD analyzer (Soken DAC-PD-9) have been used to detect and measure PDIV. Voltage was generated with a power transformer controlled with a voltage tester DACWTC-2. The equipment was calibrated before each test using a Soken DAC-CP-2 Calibration Pulse Generator. Voltage was increased with steps of $500 \mathrm{~V}$, from $0 \mathrm{~V}$ to the PDIV. The minimum charge to detect $\mathrm{PD}$ was set to $10 \mathrm{pC}$. Fig. 2 shows a schematic diagram of experimental setup for $\mathrm{PD}$ measurement.

\section{RESULTS AND DISCUSSION}

Results of the dielectric tests carried out for the oils and oilimpregnated paper are summarized in this section.

First, moisture content of the oil samples will be analyzed. FR3 moisture content was lower than 100 ppm both for fresh and aged samples, with an increase of $47 \%$ after the aging. Fresh PFAE moisture content was lower than $100 \mathrm{ppm}$. However, PFAE samples suffered an increase of the moisture content of almost $200 \%$ with the aging, despite being prepared following the same method. Since we could not measure the moisture content of the Kraft paper, PFAE was aged without

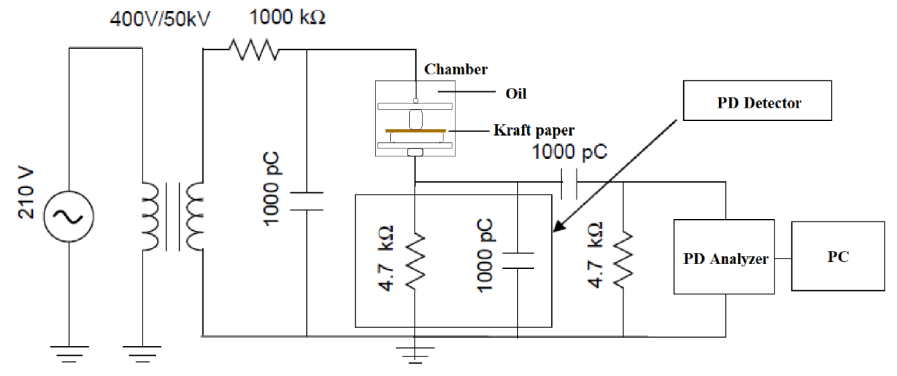

Fig. 2. Experimental setup for PD measurement. paper and the change of moisture content was lower than 59\%, which indicates that the PFAE absorbed moisture from the paper during the aging process.

Breakdown voltage results are represented in Fig. 3. As it can be seen, BDV of PFAE was higher than BDV of FR3 for every gap length. In the tests with $2.5 \mathrm{~mm}$ gap length, BDV of PFAE was higher than $100 \mathrm{kV}$, that was the maximum voltage we could applied. BDV was reduced after the aging process, up to $26 \%$ for FR3 and $30 \%$ for PFAE. Even with this larger decrease of the BDV in the case of the aged PFAE, probably due to the high moisture content, its value was higher than the one of new FR3.

Respecting relative permittivity, the results at $60 \mathrm{~Hz}$ are summarized in TABLE III. According to the data, dielectric constant of both oils was similar, and it decreased with the thermal aging.

Finally, PDIV results are collected in Fig. 4. According to other previous works [5], superficial PD have been measured with the electrode configuration used. Internal discharges have been limited due to the absence of voids between the paper sheets. PDIV was increased with the number of paper sheets for all the samples. FR3 impregnated paper had higher PDIV than the PFAE samples before the aging process, up to 24.6 $\mathrm{kV}$ for 6 paper sheets. Thermal aging effect on PDIV value was different for each oil. In the case of FR3 impregnated paper, it occurred as expected, PDIV decreased up to $8 \%$. However, with PFAE samples, no effect of aging on PDIV value was detected. It could be related to the increase in the moisture content of the oil, which could be due to the absorption of the moisture of the paper. Even with the decrease of PDIV of FR3 impregnated paper with the thermal aging, it is higher than the PFAE value in all the cases.

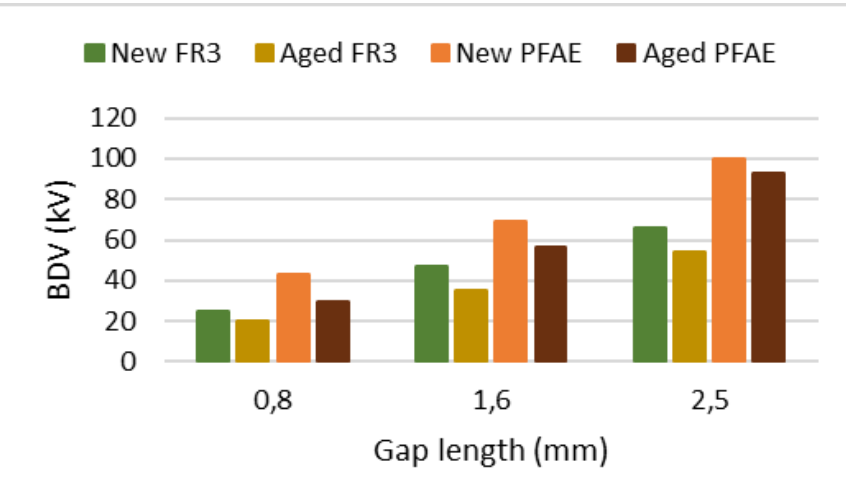

Fig. 3. BDV evolution of the oil samples.

TABLE III.

DIELECTRIC CONSTANT OF THE TESTED OILS.
\begin{tabular}{|c|c|}
\hline Sample & Dielectric constant \\
\hline New FR3 & 3.22 \\
\hline Aged FR3 & 3.18 \\
\hline New PFAE & 3.24 \\
\hline Aged PFAE & 3.2 \\
\hline
\end{tabular}




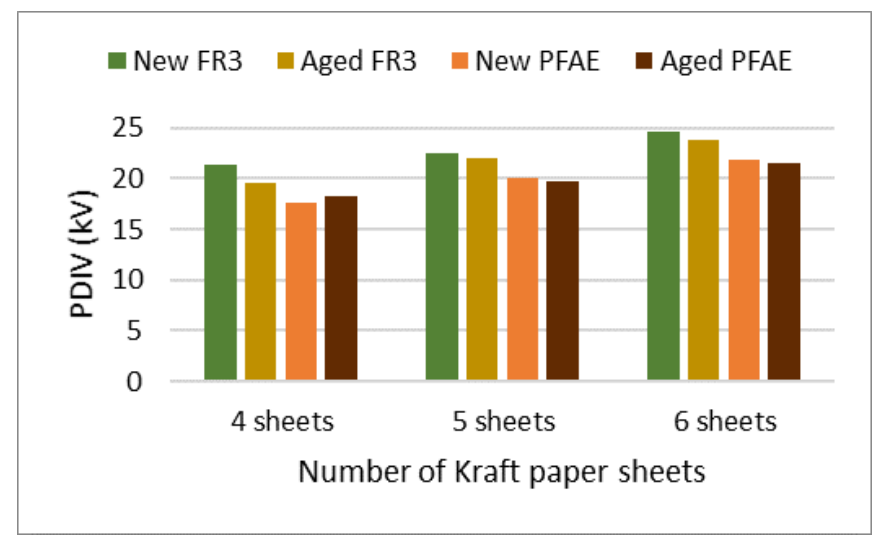

Fig. 4. PDIV dependence on thickness of paper and aging.

\section{CONCLUSIONS}

Both PFAE and FR3 were found to show good dielectric properties. BDV of the oils was studied and its evolution with the thermal aging was analyzed. PFAE had a higher BDV than new FR3 even after the thermal aging. Moisture content was lower than $100 \mathrm{ppm}$ for all the samples, except for the aged PFAE. Permittivity of both oils decreased with the aging and their values are closer. Dielectric properties of the oils in combination with Kraft paper were also studied before and after the aging process. FR3 insulating system exhibited higher PDIV than PFAE system in all the tests carried out. Thermal aging had a limited effect on PDIV value, which was smaller in the case of PFAE system in comparison with FR3 system.

Future work will be focused on the study of PD behavior at constant voltage to understand charge and electric field distribution inside the insulation system. Also, more research is needed with other biodegradable dielectric liquids and mineral oil, in order to compare their performance.

\section{ACKNOWLEDGMENT}

This research is under BIOTRAFO project, which has received funding from the European Union's Horizon 2020 Research and Innovation Program under the Marie Sklodowska-Curie Action-Research and Innovation Staff Exchange (MSCA-RISE) grant agreement No 823969.

The authors of this research wish to thank the Ministry of Economy for its financial support for the National Research Project: Improvement of Insulation Systems in Transformers Using Dielectric Nanofluids. Thermodynamic Characterization and Modelling (DPI2015-71219-C2 1-R).

Also, the authors thank the Council of Universities and Research, Environment and Social Policy for its support for the Project: Impregnation of Dielectric Solids with Biodegradable Fluids Used in Power Transformers 2019-2.

\section{REFERENCES}

[1] F. Fofana, "50 Years in the Development of Insulating Liquids," IEEE Electr. Insul. Mag., vol. 29, 2013.

[2] Q. Liu, Z. D. Wang, and F. Perrot, "Impulse breakdown voltages of ester-based transformer oils determined by using different test methods," Annu. Rep. - Conf. Electr. Insul. Dielectr. Phenomena, CEIDP, no. 1, pp. 608-612, 2009.

[3] D. Divakaran and C. Kalaivanan, "Investigation of lightning impulse voltage characteristics and other thermo-physical characteristics of vegetable oils for power apparatus applications," Proc. IEEE Int. Conf. Prop. Appl. Dielectr. Mater., pp. 1-4, 2012.

[4] J. D. ; Z. D. Wang, "A Comparison of the Impregnation of Cellulose Insulation by Ester and Mineral oil," IEEE Trans. Dielectr. Electr. Insul., vol. 15, no. 2, 2008.

[5] A. Cavallini, G. C. Montanari, and F. Ciani, "Analysis of partial discharge phenomena in paper-oil insulation systems as a basis for risk assessment evaluation," 2005 IEEE Int. Conf. Dielectr. Liq. ICDL 2005, pp. 241-244, 2005.

[6] T. A. Prevost, "Dielectric properties of natural esters and their influence on transformer insulation system design and performance - An update," 2009 IEEE Power Energy Soc. Gen. Meet. PES '09, pp. 1-7, 2009.

[7] D. Martin, Z. D. Wang, P. Dyer, A. W. Darwin, and I. R. James, "A comparative study of the dielectric strength of ester impregnated cellulose for use in large power transformers," 2007 Int. Conf. Solid Dielectr. ICSD, no. August, pp. 294-297, 2007. 